\title{
Search for gravitational waves through the electromagnetic Faraday rotation
}

\author{
M. Halilsoy* and O. Gurtug ${ }^{\dagger}$ \\ Department of Physics, Eastern Mediterranean University, G. Magusa, north Cyprus, via Mersin 10, Turkey
}

(Received 19 March 2007; published 26 June 2007)

\begin{abstract}
A method is given which renders indirect detection of strong gravitational waves possible. This is based on the reflection (collision) of a linearly polarized electromagnetic shock wave from (with) a cross polarized impulsive and shock gravitational waves in accordance with the general theory of relativity. This highly nonlinear process induces a detectable Faraday rotation in the polarization vector of the electromagnetic field.
\end{abstract}

DOI: 10.1103/PhysRevD.75.124021

PACS numbers: 04.20.- q, 02.40.-k, 04.30.Nk

\section{INTRODUCTION}

Nonlinear interaction (collision) of gravitational waves (GWs) in Einstein's theory, which was initiated a long time ago by the two letters to Nature [1,2] is a well-known subject by now $[3,4]$. As an outcome of this process we quest whether it is possible to probe controllable electromagnetic (em) waves in sensing the passing of a strong GW. Given the known exact solutions to Einstein's field equations to date, the answer is affirmative. After an encounter with a $\mathrm{GW}$, the physical changes in the em wave will inform about the latter. We recall that the first indirect evidence of GWs from the binary pulsar in the early 1970s, by the team of R. Hulse and J. Taylor also was based on em observations. The main feature on which we concentrate here is the rotation of em polarization vector known as the Faraday effect. Such rotation has been introduced also for the GWs [5-8]; however, its detection is hampered by the challenge of detecting GWs themselves.

In this paper, we propose that given its Faraday rotation after encountering with a GW, a linearly polarized em wave signals the passage of a GW. For a thorough analysis, GWs may be considered in three different profiles. The first (and simplest), albeit idealized, is an impulsive GW whose possible sources are cosmic bursts, supernova explosions, collision of black holes, etc. It is dubbed "idealized" in the sense that it arises as a limit of a Gaussian curvature. The second profile of interest is a shock type with a uniform curvature. The third type is a hybrid sandwich GW that lies intermediate to the aforementioned ones. In the sequel, we shall restrict ourselves to the two types alone.

The general form of plane waves known as the Rosen form is described by the line element

$$
\begin{aligned}
d s^{2}= & 2 e^{-M} d u d v-e^{-U}\left\{\left[e^{V} d x^{2}+e^{-V} d y^{2}\right] \cosh W\right. \\
& -2 \sinh W d x d y\} .
\end{aligned}
$$

The metric functions depend only on the null coordinates $u$ and $v$. In the plane wave regions, all metric functions appearing in this line element depend only on

\footnotetext{
*mustafa.halilsoy@emu.edu.tr
}

†ozay.gurtug@emu.edu.tr either $u$ or $v$. In this paper we shall be interested in the metric function $W(u, v)$ which represents the second polarization content of the waves.

\section{INTERACTION OF ELECTROMAGNETIC AND GRAVITATIONAL WAVES}

In our analysis, we consider the interaction of linearly polarized plane em waves with cross polarized GWs that propagate in the opposite directions in each of the incoming regions as illustrated in Fig. 1. Region III $(v>0, u<$ 0 ) contains a linearly polarized plane em wave described by the line element

$$
d s^{2}=2 d u d v-\left(\frac{1}{2}+g\right)\left(d x^{2}+d y^{2}\right),
$$

where $g=g(v)$ is only a function of $v$. The only nontrivial Einstein-Maxwell equation in this region is given by

$$
2 U_{v v}-U_{v}^{2}=4 \Phi_{00}
$$

where $e^{-U}=\frac{1}{2}+g$ and $\Phi_{00}$ stands for the Ricci tensor.

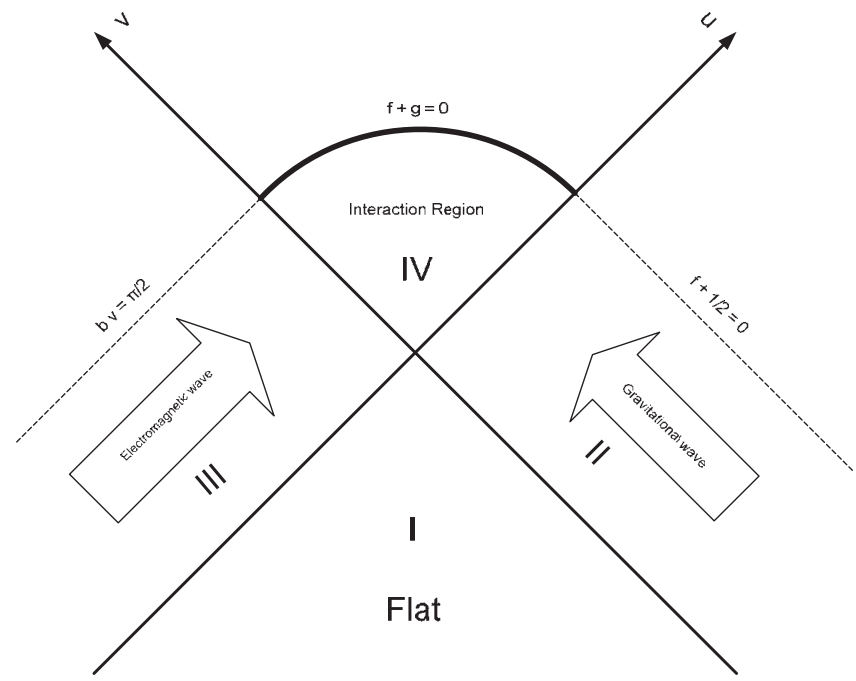

FIG. 1. The spacetime diagram describes the collision of a plane em wave propagating in one of the incoming regions and a plane GW propagating in the other incoming region. The singular hypersurface occurs when $f+g=0$ in the interaction region. 

by

Region II $(v<0, u>0)$ contains a plane GW described

$$
d s^{2}=2 d u d v-\left(\frac{1}{2}+f\right)\left(Z d \bar{x}^{2}+Z^{-1} d \bar{y}^{2}\right),
$$

in which $f$ and $Z=e^{V}$ depend only on $u$ and are constrained by the vacuum Einstein equation

$$
2 U_{u u}-U_{u}^{2}=V_{u}^{2}
$$

Now, we rotate the $(\bar{x}, \bar{y})$ axes by angle $\alpha$ to align them along with $(x, y)$ at the cost of creating a cross polarization term in (4). The two waves, em from left and GW from right, make a head-on collision on the hypersurface specified by $u=0=v$. Physically the cross $(X)$ mode of the $\mathrm{GW}$ will rotate the linear $(+)$ mode of the em wave giving rise to the Faraday effect in the em wave. Irrespective of the initial data, the Einstein-Maxwell equations admit the following metric and Maxwell fields in the interaction region $(u>0, v>0)[3]$;

$$
\begin{aligned}
d s^{2}= & \left(\frac{-g^{\prime} \sqrt{\frac{1}{2}+f}}{\sqrt{f+g} \sqrt{\frac{1}{2}-g}}\right) d u d v-(f+g)\left[\left(e^{V} d x^{2}\right.\right. \\
& \left.\left.+e^{-V} d y^{2}\right) \cosh W-2 \sinh W d x d y\right], \\
\Phi_{0}= & \left(\frac{-g^{\prime}}{2 \sqrt{f+g} \sqrt{\frac{1}{2}-g}}\right) e^{i \beta(u)}, \\
\Phi_{2}= & -\frac{\sqrt{\frac{1}{2}-g}}{2 \sqrt{f+g}}\left(V^{\prime} \cosh W+i W^{\prime}\right) e^{i \beta(u)},
\end{aligned}
$$

in which the phase function $\beta(u)$ is to be determined from $\beta^{\prime}=-\frac{1}{2} V^{\prime} \sinh W$ (a prime implies derivative with respect to the argument for each function). The incoming em shock wave with $(+)$ mode is characterized by the function

$$
g(v)=\frac{1}{2}-\sin ^{2}\left(b v_{+}\right)
$$

where $b$ is the energy (frequency) constant and $v_{+}=$ $v \theta(v)$ with $\theta(v)$ the unit step function. The initial data for the $\mathrm{GW}$ are

(i) for impulsive wave, $f=\frac{1}{2}-u_{+}^{2}$,

$$
Z=\frac{1-u_{+}}{1+u_{+}}
$$

(ii) for shock wave,

$$
\begin{aligned}
& f=-\frac{1}{2}+\cos u_{+} \cdot \cosh u_{+}, \\
& Z=\frac{\cos u_{+}}{\cosh u_{+}},
\end{aligned}
$$

where $u_{+}$is to be understood with the step function, i.e., $u \theta(u)$. The resulting solution for both cases is summarized as follows:

$$
\begin{aligned}
e^{2 V} & =\frac{Z^{2} \cos ^{2}(\alpha / 2)+\sin ^{2}(\alpha / 2)}{Z^{2} \sin ^{2}(\alpha / 2)+\cos ^{2}(\alpha / 2)}, \\
\sinh W= & \frac{1}{2}\left(Z-Z^{-1}\right) \sin \alpha, \\
\tan \beta= & \frac{1}{2 Z \cos \alpha}\left[\sqrt{4 Z^{2}+\left(1-Z^{2}\right)^{2} \sin ^{2} \alpha}\right. \\
& \left.-\left(1+Z^{2}\right) \sin \alpha\right],
\end{aligned}
$$

while $f(u)$ and $g(v)$ functions are readily available from (7) and (8). We note that $\alpha$ is confined by $0<\alpha<\pi / 2$. Before collision the em wave is linearly polarized along the $x$ axis.

\section{THE FARADAY ROTATION}

The polarization vector of the incoming em wave which was aligned with the $x$ axis does not preserve its linear form after interacting with the gravitational wave. In an orthonormal tetrad $\left(\omega^{a}\right)$, the line element can be expressed by

$$
d s^{2}=\left(\omega^{0}\right)^{2}-\left(\omega^{1}\right)^{2}-\left(\omega^{2}\right)^{2}-\left(\omega^{3}\right)^{2} .
$$

The electric and magnetic field components are defined by [9]

$$
\begin{aligned}
& E_{x}=F_{02}=\operatorname{Re}\left(\Phi_{0}-\Phi_{2}\right), \\
& H_{y}=F_{12}=-\operatorname{Re}\left(\Phi_{0}+\Phi_{2}\right), \\
& E_{y}=F_{03}=\operatorname{Im}\left(\Phi_{0}+\Phi_{2}\right), \\
& H_{x}=F_{31}=\operatorname{Im}\left(\Phi_{0}-\Phi_{2}\right) .
\end{aligned}
$$

The Faraday rotation angle $\theta$ is determined from the electric field components of the em wave by

$$
\tan \theta=\frac{\operatorname{Im}\left(\Phi_{0}+\Phi_{2}\right)}{\operatorname{Re}\left(\Phi_{0}-\Phi_{2}\right)}
$$

in which $\Phi_{0}$ and $\Phi_{2}$ are the em spinor components given in Eq. (6). In terms of metric functions, the Faraday rotation angle is

$$
\tan \theta=\frac{W^{\prime}+\left(V^{\prime} \cosh W-2 b \cot b v\right) \tan \beta}{W^{\prime} \tan \beta-V^{\prime} \cosh W-2 b \cot b v} .
$$

In the case of a gravitational plane impulsive wave and for the particular angle $\alpha=45^{\circ}$, Eq. (13) becomes

$$
\tan \theta=\left(1-u^{2}\right)\left\{\frac{1-A(u) b \cot b v}{A(u)+b\left(1-u^{2}\right)^{2} \cot b v}\right\},
$$

where $A(u)=1+u^{2}-\sqrt{2} \sqrt{1+u^{4}}$. The behavior of this expression for various $b$ values is illustrated in Fig. 2. The behavior for a shock gravitational wave case which can be obtained in analogy to Eq. (14) also from the Eqs. (8)-(13) is given in Fig. 3. Since the $v$ dependence in $\tan \theta$ is periodic, one would expect to see this behavior explicitly. 


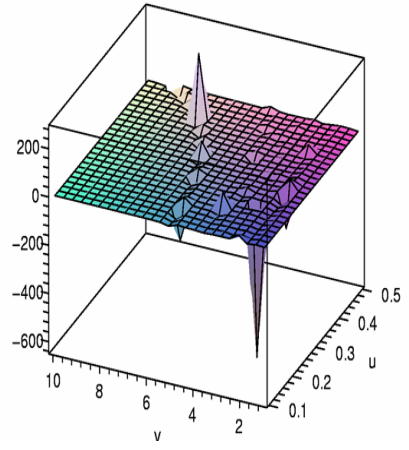

(a)

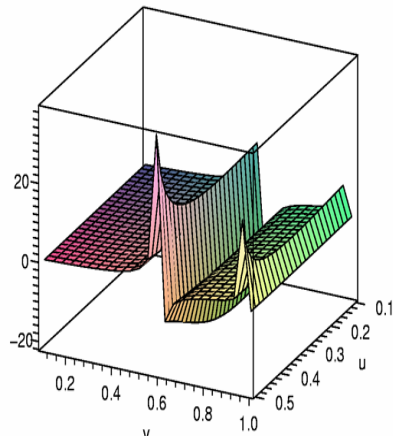

(b)

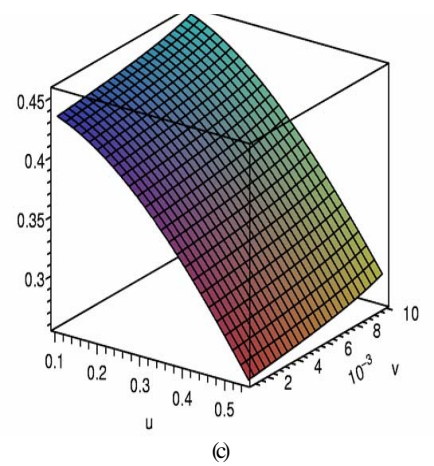

FIG. 2 (color online). The $\tan \theta$ plot when the electromagnetic shock wave encounters a gravitational plane impulsive wave with the $(X)$ polarization angle for $\alpha=45^{\circ}$, for frequencies (a) $b=0.1$, (b) $b=1$, and (c) $b=100$.

However, due to the curvature singularity that the spacetime possesses on the hypersurface, $u^{2}+\sin ^{2} b v=1$, we have to choose $0<u<1$ and $0<b v<\pi / 2$ such that $u^{2}+\sin ^{2} b v<1$, for all $(u, v)$. As a result of this constraint condition, the $v$ dependence does not display its periodic character in this range. Figures 2(a)-2(c) represent the impulsive wave plots for the parameters $b=0.1, b=1$, and $b=100$, respectively. Similarly for the shock gravitational waves, the curvature singularity occurs on the hypersurface $\cos u \cdot \cosh u+\cos ^{2} b v=1$. The values of $u$ and $v$ confined to $0<u<\pi / 2,0<b v<\pi / 2$ must be chosen in such a way to satisfy $\cos u \cdot \cosh u+\cos ^{2} b v<$ 1. The Figs. 3(a)-3(c) illustrate the shock wave plots for $b=0.1, b=1$, and $b=10$, respectively. Our study shows that highly energetic em waves (i.e. greater $b$ ) udergo rotation shortly after their encounter with the GW, while for the less energetic beams this effect is delayed. Smooth variations, beside the local extrema is another noticeable effect in different plots. One fact, however, is evident that an em wave reflecting from a cross polarized GW undergoes a Faraday rotation. Further details of this effect can be obtained by studying more plots. Our result applies also to the cosmic microwave background radiation as well as the radio astronomical fields. In other words, rotation in the polarization vector in the cosmic microwave background
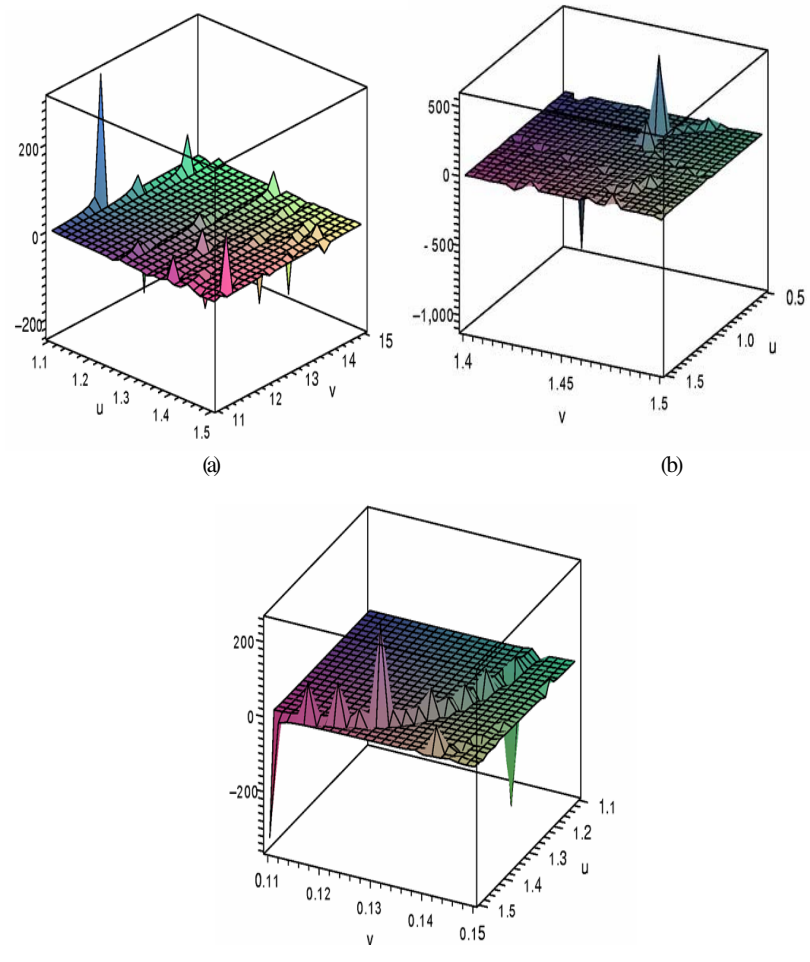

(c)

FIG. 3 (color online). The $\tan \theta$ plot when the electromagnetic shock wave encounters a gravitational shock wave with the $(X)$ polarization angle for $\alpha=45^{\circ}$, for frequencies (a) $b=0.1$, (b) $b=1$, and (c) $b=10$.

radiation can be attributed to the encounters with the strong $(X)$ moded GWs.

Considerations of mixed profile GWs and sandwich waves will be the subject of a detailed analysis. Let us remark that encountering of an em wave with a series of successive impulsive GWs has been considered already [10]. Search for GWs through the Faraday rotation due to a test em wave in a GW background has been considered by various authors $[11,12]$. Distinctly, our method takes the full nonlinear effects into account by employing the cross polarization mode of GWs.

\section{CONCLUSION}

In this paper, by using Einstein's theory of general relativity, we have analyzed the exact behavior of the polarization vector of a linearly polarized em shock wave upon encountering with GWs. As expected, the Faraday's angle emerges highly dependent on the type of the GW as well as the energy of the em wave. It suggests that compared with the polarization changes expected from the quantum process of photon-electron scattering in the early universe, or test field approximations, significant contributions to the polarization vector can be imparted by the classical em-GW collisions. 
[1] K. A. Khan and R. Penrose, Nature (London) 229, 185 (1971).

[2] P. Szekeres, Nature (London) 228, 1183 (1970).

[3] J.B. Griffiths, Colliding Plane Waves in General Relativity (Oxford University Press, Oxford, 1991).

[4] C. Barrabes and P. A. Hogan, Singular Null Hypersurfaces in General Relativity (World Scientific, Singapore, 2004).

[5] A. Tomimatsu, Gen. Relativ. Gravit.. 21, 613 (1989).

[6] M. R. Arafah, S. Fakioglu, and M. Halilsoy, Phys. Rev. D 42, 437 (1990).
[7] A. Wang, Phys. Rev. D 44, 1120 (1991).

[8] T. Piran and P. N. Safier, Nature (London) 318, 271 (1985).

[9] M. Halilsoy, J. Math. Phys. (N.Y.) 31, 2694 (1990).

[10] O. Gurtug, M. Halilsoy, and O. Unver, Phys. Rev. D 74, 044020 (2006).

[11] M. L. Rugerrio and A. Tartaglia, Mon. Not. R. Astron. Soc. 374, 847 (2007).

[12] A. M. Cruise and R. M. J. Ingley, Classical Quantum Gravity 23, 6185 (2006). 